\title{
Upper Eyelid Fistula Secondary to a Frontal Sinusitis: A Case Report and Review of the Literature
}

\author{
Laababsi Rabii*, Abdulhakeem Bushra, El Krimi Zineb, Rouadi \\ Sami, Abada Reda lah, Roubal Mohamed and Mohamed Mahtar \\ Department of Otolaryngology Head Neck Surgery, University hospital Ibn Rochd, \\ Morocco
}

\section{Case Report}

Volume 3 Issue 2

Received Date: December 10, 2018

Published Date: December 18, 2018

DOI: $10.23880 / 00 A J-16000177$

*Corresponding author: Laababsi Rabii, Department of Otolaryngology Head Neck Surgery, University hospital Ibn Rochd, Casablanca, Morocco, Tel: 00212678729101; Email: laababsirabiifmpc@gmail.com

\begin{abstract}
Introduction: Sinus disease may occur in individuals who are not otherwise aware that they have problems (occult sinusitis). An upper eyelid fistula is one of the complications of the frontal sinusitis. It can happen as a result of the obstruction of the frontal sinus ostia.

Case report: In this article, we reported a case of a 64 years old female patient admitted to our department with a 2 years' history of an upper eyelid fistula secondary to a frontal sinusitis. A paranasal sinus computed tomography (CT) concluded to a frontal and ethmoidal sinusitis associated to a frontal osteitis. The patient underwent a functional endoscopic sinus surgery to establish drainage of the affected sinuses and direct excision of the fistular tract.

Conclusion: The development of a sinocutaneous fistula secondary to a frontal sinusitis is rare, but still occurs in the modern antibiotic era. Definitive management of this disease process requires a frontal drainage of the affected sinus with fistula excision and multilayer closure.
\end{abstract}

Keywords: Frontal sinusitis; Fistula; Osteitis; Sinus surgery

\section{Introduction}

Sinus disease can present in a variety of ways and may occur in individuals who are not otherwise aware that they have problems (occult sinusitis). An upper eyelid fistula has been reported in many publications but the incidence is rare at present. Frontal sinusitis today rarely rises from a frontal abscess leading to fistula formation. This is due to a reduction in the number of cases of osteomyelitis as antibiotic use has greatly increased.
Upper eyelid fistula tends to take place as a result of the obstruction of the frontal sinus ostia by a tumor, trauma or due to defective mucociliary clearance. We report a case in which a frontal sinusitis led to an upper eyelid fistula with a delay in making the correct diagnosis. The patient was treated inappropriately before finally undergoing sinus surgery with good functional result. 


\section{Otolaryngology Open Access Journal}

\section{Clinical Case}

A 64 years old female without any particular pathological history presented to the ENT unit of our hospital with a 2 years' history of spontaneous discharge from her left upper eyelid beneath the brow ridge (Figure 1). Oral antibiotics, prescribed prior to attendance, had reduced the inflammatory signs, but not the discharge. Furthermore, the pressure on the upper eyelid caused expression of pus from the fistula. Otherwise, the patient did not have any other signs such as headache, symptoms of nasal or sinus disease, diplopia or visual problems. The lesion was drained surgically, and the patient was placed on intravenous antibiotics (third cephalosporin generation+ metronidazole) for one week with a switch to the oral form for 3 weeks. Six months later, the patient developed the same symptomatology, with a persistent supero-medial fistula. A frontal headache had occurred. A complete neurological, ophthalmological, as well as ear nose throat examination (otoscopy/rhinoscopy and nasal endoscopy) haven't found any significant abnormalities. An axial and coronal enhanced, cerebral and paranasal sinus CT revealed soft tissue opacification involving both ethmoidal sinus and left frontal sinus with left periorbital soft tissue swelling. Frontal sinus on left side showed bony dehiscence in the sinus anterior wall. Its cavity size was reduced due to thickening of the walls of the sinus. We concluded to ethmoidal and frontal sinusitis associated to a frontal osteitis (Figure 2). The lesion was biopsied to eliminate malignancy. Nasal endoscopy approach was performed under general anesthesia. During surgery, frontal sinus was exposed by drilling out anterior part of the sinus floor, and bony defect was identified from above. Sinus mucosa was appearing unhealthy. It was curetted and sent for histopathological examination. Bony sinus walls were polished with burr after removing the infected mucosa, and the frontal sinus was obliterated with abdominal fat. Upper eyelid fistula was closed in layers after excising the tract and freshening the margins of the opening. Histopathology of the sinus mucosa showed abundant granulation tissue with chronic inflammatory cells while the bone was normal. The patient was discharged after one week and she remained asymptomatic.

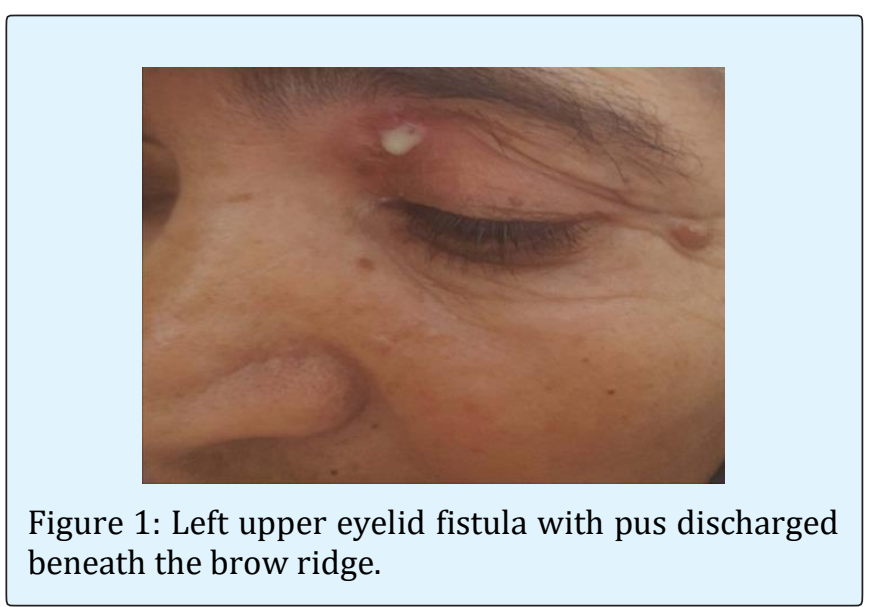

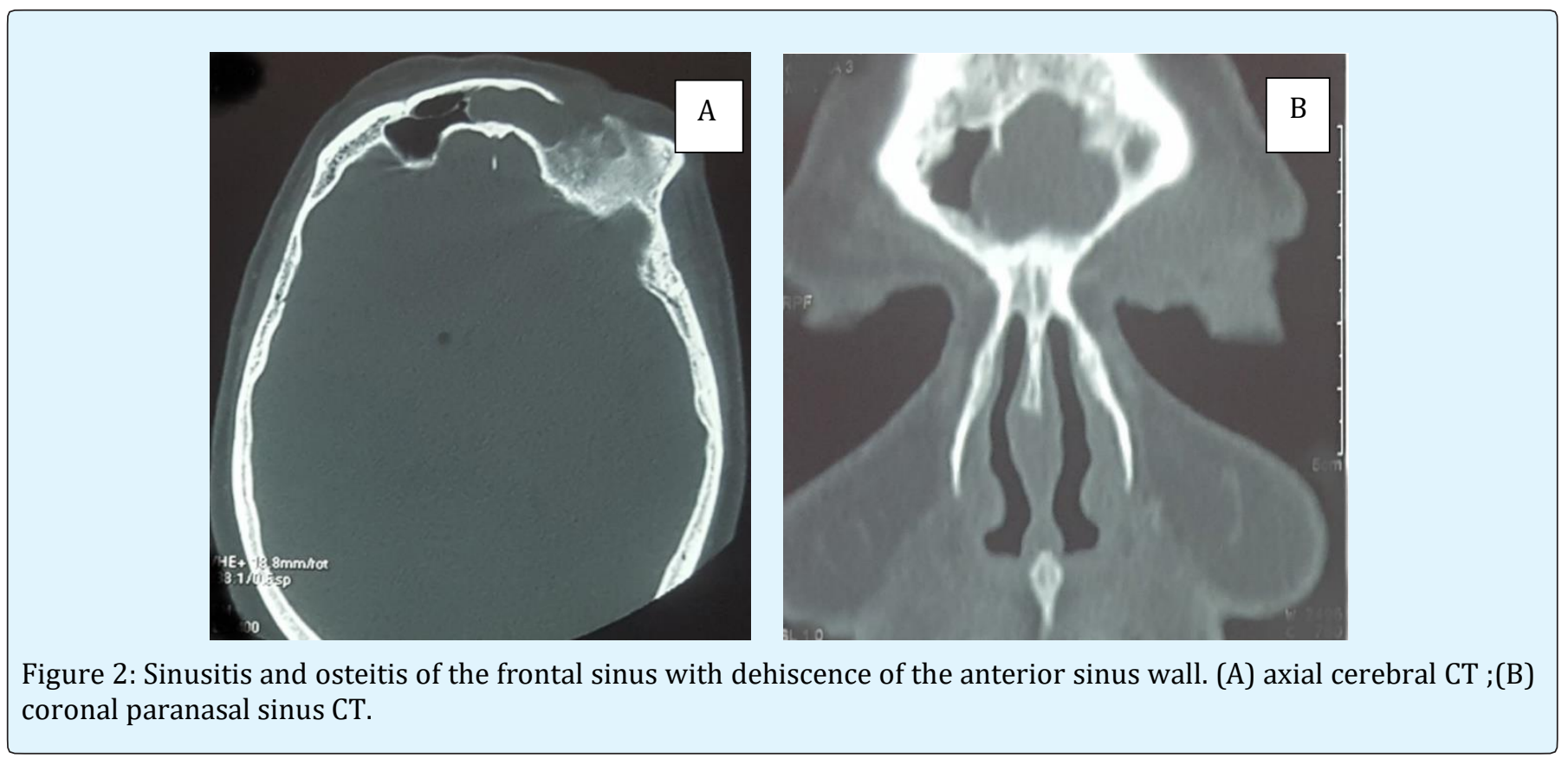

Laababsi Rabii, et al. Upper Eyelid Fistula Secondary to a Frontal Sinusitis: A Case Report and Review of the Literature. Otolaryngol Open Access J 2018, 3(2): 000177. 


\section{Otolaryngology Open Access Journal}

\section{Discussion}

A frontal sinocutaneous fistula develops as a communication from the wall of the frontal sinus to the overlying skin. Such a lesion can be the result of osteomyelitis, bone necrosis and erosion due to a mucocele, tumor or bone traumatic injury [1].

In the study by $\mathrm{Wu}$, et al. out of the 3 patients, one presented with a left forehead fistula, another had a midline forehead fistula, while the last case had a left upper eyelid fistula. In all cases, the anterior wall of the frontal sinus was found breached. All patients had a history of frontal sinusitis [1].

Marshall and Jones describe a review where out of 7 cases of osteomyelitis, 4 had frontal swelling with forehead fistula [2]. Marfatia, et al. reported a case of a persistently discharging fistula on the left side of forehead, which had a defect in both anterior and posterior walls of frontal sinus with history of frontal sinusitis [3].

Seyhan and Ozerdem describe a case of a 21-year-old man with an upper lid abscess who was treated with several courses of oral antibiotics [4]. Following each course, the abscess would settle and then recur after 4 to 5 months. He developed a continuous discharge and, finally, after 3 years the correct diagnosis of a frontal sinus fistula was made using CT. Casady, et al. describe a series of 5 patients who initially presented with upper eyelid abscesses, but following investigation were later found to have sinus related fistula [5]. Another more recent retrospective case series from Moorfields Eye Hospital describes features of 8 patients presenting with eyelid fistula due to chronic sinus disease [6]. All presented with discharge from the fistula which was situated either centrally or medially in the upper lid. In addition, three of the patients had associated ptosis, two had proptosis, and one had a medial upper lid ectropion. All had opacification of their ipsilateral frontal or ethmoidal sinus on CT imaging; five showed bony erosion and two had sinus mucocoeles. Secondary fistulous communication to the upper lid is relatively uncommon but should be suspected if an individual presents with a chronic upper lid abscess that does not appear to settle and imaging with CT scanning should be considered at an early stage to investigate the extension of all persisting abscesses. Failure to recognize this diagnosis can expose the patient to other more serious and more well-known risks of sinus disease, such as cavenous sinus thrombosis and orbital cellulitis.
Endoscopic sinus surgery remains the gold standard for the treatment of chronic sinusitis [7], it appears to be a very effective method for the treatment of an upper eyelid fistula when it combined with excision of the fistular tract. Endoscopic surgery attempts to restore normal ciliary flow within the sinuses using minimal invasive surgery on the osteo-meatal unit [8], and can usually establish intranasal drainage of mucocoeles [9]. In contrast, traditional surgical approaches emphasize the drainage of affected sinuses with extensive removal of inflamed mucosa [8].

\section{Conclusion}

Development of a sinocutaneous fistula secondary to frontal sinusitis is rare, but still occurs in the modern antibiotic era. Definitive management of this disease process requires a frontal drainage of the affected sinus with fistula excision and multilayer closure to avoid any complications that can be more dangerous.

\section{Conflict of interest: None.}

Informed Consent: The patient gave us informed consent for publication.

\section{References}

1. Wu VF, Smith TL, Poetker DM (2008) Sinocutaneous fistula secondary to chronic frontal rhinosinusitis: Case series and literature review. Ann Otol 117(10): 759-763.

2. Marshall AH, Jones NS (2000) Osteomyelitis of the frontal bone secondary to frontal sinusitis. J Laryngol Otol 114(12): 944-946.

3. Marfatia HK, Muranjan SN, Navalakhe MM, Kirtane MV (1997) Persistent frontal fistula. J Postgrad Med 43(4): 102-103.

4. Seyhan T, Ozerdem OR (2005) Upper eyelid fistula caused by chronic frontal sinusitis. J Craniofac Surg 16(1): 171-174.

5. Casady DR, Zobal-Ratner JL, Meyer DR (2005) Eyelid abscess as a presenting sign of occult sinusitis. Ophthal Plast Reconstr Surg 21(5): 368-370.

6. Rossman D, Verity DH, Lund VJ, Rose GE (2007) Eyelid fistula: a feature of occult sinus disease. Orbit 26(3): 159-163. 


\section{Otolaryngology Open Access Journal}

7. Videler WJM, Wreesmann VB, Van Der Meulen FW, Knegt PP, Fokkens WJ (2006) Repetitive endoscopic sinus surgery failure: A role for radical surgery? Otolaryngol Head Neck Surg 134(4): 586-591.

8. Kennedy DW, Zinreich SJ, Rosenbaum AE, Johns ME (1985) Functional endoscopic sinus surgery, Theory and diagnostic evaluation. Arch Otolaryngol 111(9): 576-582.

9. Serrano E, Klossek JM, Percodani J, Yardeni E, Dufour $X$ (2004) Surgical management of paranasal sinus mucoceles: A long-term study of 60 cases. Otolaryngol Head Neck Surg 131(1): 133-140. 